\title{
Gastrointestinal injurious insults in gynecological surgical procedures at EI Sahel Hospital
}

Gynecology and Women's Health Care

Research Article

\author{
Amr Abu Ella $^{1 *}$, Abdelrahman Kamal Abdelrahman ${ }^{1,}$ Shaimaa Belal ${ }^{2}$ and Manal Abdelwanees Elsayed ${ }^{3}$ \\ ${ }^{1}$ Department of General Surgery, El Sahel Teaching Hospital. Cairo. Egypt \\ ${ }^{2}$ Department of Obstetrics and Gynecology, Helwan University. Helwan. Egypt \\ ${ }^{3}$ Department of Obstetrics and Gynecology, El Sahel Teaching Hospital. Cairo. Egypt

$\begin{array}{ll}\text { Submitted } & \text { : January } 8^{\text {th }}, 2020 \\ \text { Accepted } & : \text { January } 14^{\text {th }}, 2020 \\ \text { Published } & : \text { January } 20^{\text {th }}, 2020\end{array}$ \\ Correspondence author \\ Dr Abdelrahman Kamel Abdelrahman \\ Department of General Surgery \\ El Sahel Teaching Hospital \\ Cairo, Egypt \\ Tel : $\quad 01008768899$ \\ E-mail : elnagarw581@gmail.com
}

\begin{abstract}
Background: Gastrointestinal surgical insults during operative gynecologic procedures varies considerably according to the procedure. Prognostic tools and risk factors should be elucidated by further research efforts to enhance management levels of those cases category.

Aim of the work: To investigate the risk factors, impact and severity as regards gastrointestinal injuries during gynecological and obstetrical operations.

Methodology: A retrospective case-control research study performed on cases who have underwent gynecologic surgery in the period between 2014 and 2019.

Results: Risk factors for gastrointestinal injuries in which past history of abdominal operation, pelvic adhesions, previous pelvic operation have shown to be statistically significant risk factors for GIT injuries ( $p$ values $<0.001$ ),furthermore ovarian malignancy and staging have been statically significant risk factors affecting the occurrence of GIT injuries in gynecological procedures ( $p$ values $=0.007,0.019$, consecutively)

Conclusions and Recommendations: Innovation of a surgical prognosis algorithm based on statistical analysis is considered the cornerstone of enhancement of surgical management of gynecologically challenging cases aiding in reducing gastrointestinal surgical insults within gynecological surgical practice.
\end{abstract}

\section{Introduction}

Gastrointestinal injuries during gynecological operations whether open or laparoscopically is a challenging clinical case scenario necessitating proper diagnostic protocol to avoid delayed diagnosis and arousal of morbidity and mortality issues [1].

The incidence of intestinal injuries varies considerably according to the procedure whether dilatation and curettage and trocar injuries. Simple repair by primary closure is considered enough in many case scenarios however sometimes there is more extensive injuries requiring resection and anastomosis that is well known to be a lifesaving procedure [2].

On the other hand, a comprehensive and detailed knowledge of gastrointestinal anatomy with expected adhesions in cases

with prior surgical interventions could raise the surgeon's meticulousness to and cautiousness in avoidance of iatrogenic injuries to the gastrointestinal tract. Prognostic tools for gastrointestinal injuries are technique and the type of surgery, prior gastrointestinal and /or pelvic surgery. Cases having a prior history of diverticulitis and pelvic inflammatory disease may have raised risk for intraoperative iatrogenic intestinal injuries [3].

Small intestinal injuries occur in around $75 \%$ of cases, whereas $25 \%$ of cases injuries affect the large intestine and rarely within gastric area. Interestingly research data available about medical and surgical risk factors predisposing to gastrointestinal injuries are scarce [4]. 


\section{Aim of the work}

To investigate the risk factors, impact and severity as regards gastrointestinal injuries during gynecological and obstetrical operations.

\section{Methodology}

A retrospective case-control research performed on cases who have underwent gynecologic surgery from 2014 to 2019 . A gastrointestinal injury has been defined as an avulsion, laceration, incision, transection or a thermal insult to viscera diagnosed intraoperatively or suspected by imaging investigations within post-operative period. The data correlated to clinical outcome has been recorded, e.g. injury site, diagnosis time, repair technique. Research data have been obtained from the medical recording system, e.g. age, body mass index (BMI), surgical history, infection of the pelvis, adhesions, surgical technique of operations performed, how much blood loss and the hospital admission period.

\section{Statistical analysis}

Data have been collected, checked, coded and assembled to the statistical package for social science (SPSS) Version 23. Qualitative data have been assembled as numbers and percentages and compared by Chi-square test and/or Fisher exact test only if the expected count found less than [5]. The quantitative data have been presented as mean, standard deviations and ranges for parametric data and compared by using Independent t-test plus median with inter-quartile range (IQR) for non-parametric data and compared using Mann-Whitney test. Univariate logistic regression analysis has been used to assess the independent risk factors of GIT injuries during gynecologic operations followed by multivariate analysis using backward (Wald) method. The confidence interval has been set to $95 \%$ and the error margin accepted has been set to $5 \%$. Hence, the p-value has been considered significant at the level of $<0.05$.

\section{Results}

\begin{tabular}{|c|c|c|c|c|c|c|}
\hline & \multicolumn{2}{|c|}{$\begin{array}{c}\text { Small } \\
\text { intestine }\end{array}$} & \multicolumn{2}{|c|}{$\begin{array}{l}\text { Large } \\
\text { intestine }\end{array}$} & \multicolumn{2}{|c|}{ Rectum } \\
\hline & No. & $\%$ & No. & $\%$ & No. & $\%$ \\
\hline \multicolumn{7}{|l|}{ Open surgery } \\
\hline $\begin{array}{l}\text { Transabdominal hysterectomy or } \\
\text { myomectomy }\end{array}$ & 2 & $4.0 \%$ & 1 & $2.0 \%$ & 3 & $6.0 \%$ \\
\hline $\begin{array}{l}\text { Transabdominal hysterectomy with } \\
\text { adnexal operation }\end{array}$ & 9 & $18.0 \%$ & 6 & $12.0 \%$ & 4 & $8.0 \%$ \\
\hline Adnexal operation & 3 & $6.0 \%$ & 2 & $4.0 \%$ & 2 & $4.0 \%$ \\
\hline Surgical staging & 7 & $14.0 \%$ & 6 & $12.0 \%$ & 3 & $6.0 \%$ \\
\hline \multicolumn{7}{|l|}{ Laparoscopic surgery } \\
\hline Hysterectomy or myomectomy & 0 & $0.0 \%$ & 0 & $0.0 \%$ & 0 & $0.0 \%$ \\
\hline Hysterectomy with adnexal operation & 1 & $2.0 \%$ & 1 & $2.0 \%$ & 0 & $0.0 \%$ \\
\hline Adnexal operation & 0 & $0.0 \%$ & 0 & $0.0 \%$ & 0 & $0.0 \%$ \\
\hline Total & 22 & $44 \%$ & 16 & $32 \%$ & 12 & $24 \%$ \\
\hline
\end{tabular}


Table 1 : Type of gynecological procedure in patients with gastrointestinal injuries

Table (1) displays the category of gynecological procedures performed among cases having gastrointestinal injuries in which transabdominal hysterectomy or myomectomy 2 cases had small bowel injuries, 1 case had colonic injury, and 3 cases had rectal injuries, whereas transabdominal hysterectomy with adnexal operation 9 cases had small bowel injuries, 6 cases had colonic injuries, 4 cases had rectal injuries, furthermore adnexal procedures performed 3 cases had small bowel injuries, 2 cases had colonic injuries and 2 cases had rectal injuries, surgical staging procedures involved 7 cases with small intestinal injuries, 6 cases had colonic injuries, 3 cases rectal injuries.

As regards laparoscopic procedures only 1 case of small bowel injury and 1 case of colonic bowel injury in hysterectomy with adnexal operation. Totally 22 cases had small intestinal injuries representing $44 \%, 16$ cases had colonic injuries representing 32 $\%$, and 12 cases had rectal injuries representing $24 \%$ of cases with gastrointestinal injuries.

Table 2 : Demographic data of cases and control research groups

\begin{tabular}{|l|l|l|l|l|l|}
\hline & Cases group & Control group & Test value & \multirow{2}{*}{ P-value } & \multirow{2}{*}{ Sig. } \\
\cline { 2 - 3 } & No. $=\mathbf{5 0}$ & No. $=\mathbf{2 0 0}$ & & & \\
\hline Ag in years, mean \pm SD & $45.3 \pm 9.6$ & $43.8 \pm 8.4$ & $1.097^{*}$ & 0.273 & NS \\
\hline BMI, mean \pm SD & $24.2 \pm 2.3$ & $23.89 \pm 2.4$ & $0.824^{*}$ & 0.411 & NS \\
\hline Parity, median $(\mathrm{IQR})$ & $2(1-3)$ & $1(1-3)$ & 1.085 & 0.341 & NS \\
\hline Mass size $(\mathrm{cm})$, mean \pm SD & $9.4 \pm 3.5$ & $10.1 \pm 2.4$ & $1.668^{*}$ & 0.097 & NS \\
\hline Previous history of PID & $1(2.0 \%)$ & $6(3 \%)$ & $0.147 \ddagger$ & 0.701 & NS \\
\hline Approach of surgery & & & $1.276 \ddagger$ & 0.258 & NS \\
\hline Open & $48(96.0 \%)$ & $197(98.5 \%)$ & & & \\
\hline Laparoscope & $2(4.0 \%)$ & $3(1.5 \%)$ & & & \\
\hline EBL $(m)$, Median $(\mathrm{IQR})$ & $485(370-715)$ & $225(130-450)$ & 4.314 & $<0.001$ & HS \\
\hline LOS, Median $(\mathrm{IQR})$ & $7(5-9)$ & $5(4-6)$ & 2.584 & 0.009 & $\mathrm{~S}$ \\
\hline
\end{tabular}

*: Independent t-test; $\bullet:$ Mann-Whitney test; $\$$ : Chi-square test LOS: Length of stay

Table (2) reveals and displays that the total cohort involved 50 cases and 200 control research groups in which age, body mass index (BMI), parity, size of the mass, prior history of pelvic inflammatory disease (PID), surgical approach ( $\mathrm{p}$ values $=0.273$, $0.411,0.341,0.097,0.701,0.258$, consecutively) on the other hand estimated blood loss (EBL), length of hospital stay (LOS) have been statistically significantly higher in case group ( $\mathrm{p}$ values $<0.001,0.009$ consecutively). 
Table 3: Risk factors for gastrointestinal injuries during gynecological operations

\begin{tabular}{|c|c|c|c|c|c|c|c|}
\hline & \multicolumn{2}{|c|}{ Cases group } & \multicolumn{2}{|c|}{$\begin{array}{l}\text { Control } \\
\text { group }\end{array}$} & \multirow[t]{2}{*}{ P-value* } & \multicolumn{2}{|l|}{ OR (95\% CI), p-value } \\
\hline & No. & $\%$ & No. & $\%$ & & Univariate, & Multivariate \\
\hline Previous abdominal surgery & 9 & $18.0 \%$ & 5 & $2.5 \%$ & $<0.001$ & $\begin{array}{l}8.561(2.7272 \text { to } 26.8734), \\
<0.001\end{array}$ & $\begin{array}{l}7.2(1.885 \\
-27.495) \\
<0.001\end{array}$ \\
\hline Pelvic adhesion, & 41 & $82.0 \%$ & 57 & $28.5 \%$ & $<0.001$ & $\begin{array}{l}11.428(5.2177 \text { to } 25.0340) \\
<0.001\end{array}$ & $\begin{array}{l}40.8(15.038 \\
-110.692), \\
<0.001\end{array}$ \\
\hline Previous pelvic surgery & 23 & $46.0 \%$ & 12 & $6.0 \%$ & $<0.001$ & $\begin{array}{l}13.346(5.9598 \text { to } 29.8845) \\
<0.001\end{array}$ & \\
\hline \multicolumn{8}{|l|}{ Diagnosis } \\
\hline Ovarian cancer & 16 & $32.0 \%$ & 30 & $15.0 \%$ & 0.005 & 2.667 (1.3114 to 5.4226), 0.007 & \\
\hline Endometrial cancer & 2 & $4.0 \%$ & 6 & $3.0 \%$ & 0.719 & $\begin{array}{l}1.3472(0.2636 \text { to } 6.8845) \\
0.720\end{array}$ & \\
\hline Cervical cancer & 1 & $2.0 \%$ & 1 & $0.5 \%$ & 0.286 & $\begin{array}{l}4.0612 \text { (0.2496 to } 66.0830), \\
0.325\end{array}$ & \\
\hline Adenomyosis & 6 & $12.0 \%$ & 29 & $14.5 \%$ & 0.648 & $\begin{array}{l}0.8041 \text { ( } 0.3143 \text { to } 2.0572) \\
0.649\end{array}$ & \\
\hline Endometriosis & 12 & $24.0 \%$ & 29 & $14.5 \%$ & 0.104 & $\begin{array}{l}1.8621 \text { ( } 0.8717 \text { to } 3.9779), \\
0.108\end{array}$ & \\
\hline Ovarian mass & 8 & $16.0 \%$ & 28 & $14.0 \%$ & 0.718 & $\begin{array}{l}1.1701(0.4976 \text { to } 2.7516), \\
0.719\end{array}$ & \\
\hline Uterine fibroid & 11 & $22.0 \%$ & 60 & $30.0 \%$ & 0.261 & $\begin{array}{l}0.6581(0.3158 \text { to } 1.3715), \\
0.264\end{array}$ & \\
\hline \multicolumn{8}{|l|}{ Type of operation } \\
\hline Surgical staging & 17 & $34.0 \%$ & 37 & $18.5 \%$ & 0.017 & $\begin{array}{l}2.2695 \text { (1.1435 to } 4.5039) \\
0.019\end{array}$ & $\begin{array}{l}0.104(0.036- \\
0.304),<0.001\end{array}$ \\
\hline Hysterectomy with SO & 20 & $40.0 \%$ & 62 & $31.0 \%$ & 0.225 & $\begin{array}{l}1.4839(0.7822 \text { to } 2.8149), \\
0.227\end{array}$ & \\
\hline Hysterectomy & 6 & $12.0 \%$ & 45 & $22.5 \%$ & 0.099 & $\begin{array}{l}0.4697(0.1881 \text { to } 1.1731) \\
0.106\end{array}$ & \\
\hline Myomectomy & 0 & $0.0 \%$ & 12 & $6.0 \%$ & 0.075 & $\begin{array}{l}0.1493(0.0087 \text { to } 2.5651), \\
0.189\end{array}$ & \\
\hline $\mathrm{SO}$ & 6 & $12.0 \%$ & 13 & $6.5 \%$ & 0.189 & $\begin{array}{l}1.9615(0.7062 \text { to } 5.4483), \\
0.196\end{array}$ & \\
\hline Cystectomy & 4 & $8.0 \%$ & 20 & $10.0 \%$ & 0.667 & $\begin{array}{l}1.9615(0.2550 \text { to } 2.4017), \\
0.668\end{array}$ & \\
\hline
\end{tabular}

\section{*: P-value of Chi-square test}

Table (3) reveals and displays the risk factors for GIT injuries in which prior abdominal surgery, pelvic adhesions and prior pelvic operation have shown to be statistically significant risk factors for GIT injuries ( $p$ values $<0.001$ ), furthermore ovarian malignancy and staging have been statically significant risk factors affecting the occurrence of GIT injuries in gynecological procedures ( $\mathrm{p}$ values $=0.007,0.019$, consecutively).

\section{Discussion}

Gastrointestinal surgical insults during operative gynecologic procedures are not common; however, it could cause considerable morbidity and mortality issues. Late diagnosis of GIT injury could end with abdominal pain, nausea, hyperpyrexia, sepsis or death by a mortality rate of $21 \%$. A prior research study, the 10 -year GI injuries incidence in gynecologic surgery have been estimated 
to be around $0.38 \%$. The cornerstone risk factors correlated to bowel injury was prior abdominal surgery, pelvic adhesions. The only approach to reduce and minimize gastrointestinal iatrogenic injuries and avoid missed injuries is the meticulous abdominal examination, gentle tissue dissection and prediction of elevated risk level in cases with prior abdominal operative interventions. Minimal invasive gynecological procedures have been more prevalent with better precise techniques and instrument utilization to avoid occult visceral iatrogenic injurious insults $[5,6]$.

Intraabdominal incidence of adhesion after the open operation have been statistically estimated from prior research studies to be around 30 to $90 \%$ furthermore intestinal injuries were observed to occur in around $13.4 \%$ of cases having gynecologic laparoscopy with history of laparotomy. The requirement for surgical performance of adhesiolysis could justify the cause for the high injury rate observed (furthermore difficult approach of the abdominal cavity, especially in previous abdominopelvic surgical interventions). Also, the adhesions between the scar and underlying viscera are an adverse event of previous abdominal surgery $[7,8]$.

A prior descriptive retrospective research study, in which 25 cases with gastrointestinal injuries during gynecological procedures research data was statistically analyzed the research team of linvestigators revealed and displayed that the mean $+/$-SD age of cases $=33.2 \pm 7.57$ yrs. $44 \%$ of research study subjects recruited had an abdominal wall scar. $32 \%$ of all gastrointestinal surgical injuries happened while performance of total abdominal hysterectomy, the small intestine has been injured in $36 \%$ of cases. $52 \%$ of injuries have been successfully detected intraoperatively and the mean+/-SD diagnosis time of injury $=2.8 \pm 0.9$ days. The research investigators observed the conclusion that all gynecologists should be aware of GIT injuries and expect injury to those organs, particularly in surgically challenging high-risk cases to reduce morbidity issues among those categories of cases $[9,10]$.

Furthermore, prior clinical research studies, GIT injury incidence while doing gynecologic surgery was in between $0.05 \%$ and $0.7 \%$, another research have shown that occurrence of intestinal injury because of laparoscopy was about $(0.05 \%)$ like the current research study results. Interestingly prior researchers have observed that gastrointestinal injuries have occurred less frequently in laparoscopic surgery than in open surgical procedures ( $98 \%$ versus $2 \%$ ), those results could be justified that surgeons could convert from laparoscopic surgery to open surgery in surgical scenarios of extensive adhesions and less probability of intestinal manipulation while using laparoscopy than in open surgery. Interestingly it was observed by various research studies previously performed that the gastrointestinal injury site relies on the operation type [11, 12].

A similar study to the current one in approach and methodology have assessed that the most frequent injury site in the bowel have been the small intestine $(43.3 \%)$, which shows great similarity to the current study results. Additionally, it has been observed and displayed that most surgical injuries have been minor. Interestingly speedy GI injury diagnosis within intraoperative time period carries good prognosis when corrective management is performed without delay [13].

Interestingly prior researchers have observed in a considerable fashion that the incision type had a cornerstone in gastrointestinal surgical insults. History of abdominal surgery raised the probability of gastrointestinal surgical insults more than past pelvic surgery. Advanced age, endometriosis disease, malignant ovarian diseases and staging have been similarly observed in prior research studies to be risk factors for gastrointestinal surgical insults similar to the findings of the current research study. It is crucial to mention that endometriosis and adhesion have a mutual anatomical manipulating feature causing tissue plane alteration making adhesiolysis a risky intervention even when required intraoperatively $[3,12]$.

\section{Conclusions and Recommendations}

Innovation of a surgical prognosis algorithm based on statistical analysis is considered the cornerstone of enhancement of surgical management of gynecologically challenging cases aiding in reducing gastrointestinal surgical insults within gynecological surgical practice.

\section{References}

1. Burbos N, Abu-Friej M, Kapur S, Simon G Crocker, Timothy J Duncan, et al. (2011) How to improve training in bowel surgery for gynecological oncologists-experience from a single center in the United kingdom. Int J Gynecol Cancer 21: 1692-Y1694.

2. Peiretti M, Bristow RE, Zapardiel I (2012) Recto sigmoid resection at the time of primary cytoreduction for advanced ovarian cancer. A multi-center analysis of surgical and oncological outcomes. Gynecol Oncol 126: 220-Y223.

3. Aviki EM, Rauh-Hain JA, Clark RM, Hall TR, Berkowitz LR, et al. (2015) Gynecologic oncologist as surgical consultant: intraoperative consultations during general gynecologic surgery as an important focus of gynecologic oncology training. Gynecol Oncol 137: 93-Y97.

4. Naik R, Galaal K, Alagoda B (2010) Surgical training ingastrointestinal procedures within a UK gynaecological oncology subspecialty programme. BJOG 117: 26-Y31.

5. Barton DP, Bridges JE, Ind TE (2010) Surgical training in gastrointestinal procedures within a UK gynaecological oncology sub specialty programme. BJOG 117: 768Y769.

6. Higgins RV (2016) The Gynecologic Oncology Milestone Project. The Accreditation Council for Graduate Medical Education, the American Board of Obstetrics and Gynecology, and the American College of Obstetricians 
and Gynecologists.

7. Ciotti M, Johnson MJ (2012) Members of the Review Committee for Obstetrics and Gynecology, Minimum Thresholds for Obstetrics and Gynecology Procedures. Accreditation Council for Graduate Medical Education. http://www.acgme.org.

8. Common Program Requirements (2019) ACGME Program Requirements for Graduate Medical Education in Gynecologic Oncology (Subspecialty of Obstetrics and Gynecology). Accreditation Council for Graduate Medical Education 2019: 1-54.

9. Khoury W, Abu-Abeid S, Person B, Klausner JM, Kariv Y (2012) Missed inadvertent gastrointestinal injuries during abdominal operations: characteristics, diagnosis, and treatment. The American Surgeon 78: 46-50.

10. Kumakiri J, Kikuchi I, Kitade M, Kuroda K, Matsuoka S, et al. (2010) Incidence of complications during gynecologic laparoscopic surgery in patients after previous laparotomy. Journal of Minimally Invasive Gynecology 17: 480-486.

11. Mesdaghinia E, Abedzadeh-Kalahroudi M, Hedayati M, Moussavi-Bioki N (2013) Iatrogenic gastrointestinal injuries during obstetrical and gynecological operation. Archives of Trauma Research 2: 81-84.

12. Manchanda R, Godfrey M, Wong-Taylor LA, MJ Halaska, M Burnell, et al. (2013) The need for accredited training in gynaecological oncology: a report from the European Network of Young Gynaecological Oncologists (ENYGO). Ann Oncol 24: 944-Y952.

13. 13. Mowat A, Maher C, Ballard E (2016) Surgical outcomes for low-volume vs high-volume surgeons in gynecology surgery: a systematic review and metaanalysis. Am J Obstet Gynecol 215: 21-Y33. 